\title{
The Pup-Proteasome System of Mycobacteria
}

\author{
Nadine J. Bode and K. Heran Darwin \\ New York University School of Medicine, Department of Microbiology, 550 First Avenue, MSB \\ 236, New York, NY 10016
}

\begin{abstract}
Proteasomes are ATP-dependent, barrel-shaped proteases found in all three domains of life. In eukaryotes, proteins are typically targeted for degradation by posttranslational modification with the small protein ubiquitin. In 2008, the first bacterial protein modifier, Pup (prokaryotic ubiquitin-like protein) was identified in Mycobacterium tuberculosis (Mtb). Functionally analogous to ubiquitin, conjugation with Pup serves as a signal for degradation by the mycobacterial proteasome. Proteolysis-dependent and -independent functions of the $M t b$ proteasome are essential for virulence of this successful pathogen. In this chapter we describe the discovery of the proteasome as a key player in tuberculosis pathogenesis and the biology and biochemistry of the Pup-proteasome system.
\end{abstract}

Murine models of tuberculosis have implicated the production of nitric oxide (NO) by activated macrophages as a pivotal part of the immune response, as mice lacking inducible nitric oxide synthase (iNOS ${ }^{--}$) readily succumb to infection with $M t b$ (1). Formation of reactive nitrogen and oxygen intermediates (RNI and ROI) is toxic to a variety of microbes (reviewed in 2). The free radical NO is neutral and hydrophobic, allowing it to pass cellular and bacterial membranes. Reaction with superoxide generated by NADPH phagocyte oxidase results in the formation of the particularly destructive product peroxynitrite. The cytotoxic effects of RNI and ROI include DNA strand breakage, lipid peroxidation and protein damage (reviewed in 3,4,5). Although the importance of NO has not yet been irrevocably demonstrated in humans, several in vitro studies suggest a role of host-derived RNI in control of tuberculosis (reviewed in 2,6).

In an effort to deepen the understanding of the bacterial mechanisms in place to avoid elimination by the host immune system, Darwin et al. performed a genetic screen to discover genes that confer resistance to RNI in vitro. After screening 10,100 transposon mutants of H37Rv for hypersensitivity to acidified nitrite, the authors identified five mutants with independent insertions in Rv2097c and Rv2115c, two genes encoding putative accessory factors of a protease called the proteasome (7). Rv2115c was predicted to encode a AAA ( ATPase associated with various cellular activites) ATPase related to those associated with the proteasome in eukaryotes. On the basis of its genomic location and a high degree of sequence similarity $(81 \%)$ to ARC (AAA ATPase forming Ring-shaped Complexes) from Rhodococcus erythropolis, the authors named Rv2115c mpa 
(mycobacterial proteasome ATPase). ARC was the first characterized bacterial ATPase with suggested proteasomal function (8). In contrast, Rv2097c was annotated as a hypothetical protein (9) and shared no homology with any protein of known function. Mutations in Rv2097c and mpa caused similar phenotypes in vivo and in vitro, suggesting that both gene products participate in the same pathway. As a result of this observation, as well as its genomic association with the proteasome core protease-encoding prcBA genes, Rv2097c was named pafA for proteasome accessory factor $\underline{\mathrm{A}}$ (7).

The identification of proteasomal components as potential anti-tuberculosis targets that would sensitize $M t b$ to host immunity immediately raised the interest of the scientific community. Previously, no function had been assigned to the bacterial proteasome and attempts to reconstitute protein degradation by a bacterial proteasome in vitro had proven unsuccessful (8), likely due to the lack of additional required cofactors. Over the course of the last decade, seminal experiments demonstrated the existence of a bacterial posttranslational protein modifier called Pup (prokaryotic ubiquitin-like protein), which serves as a signal for degradation by the mycobacterial proteasome. Here we summarize what is currently known about the genetics and function of the $M t b$ Pup-proteasome system and its role in pathogenesis.

\section{Prokaryotic proteasomes}

Proteasomes are self-compartmentalizing, ATP-dependent multi-subunit proteases that are responsible for the majority of non-lysosomal protein degradation in eukaryotes. The barrelshaped complexes have critical function in protein quality control, stress response, cell cycle control, transcription, metabolism, signal transduction, immune response and many other biological processes (reviewed in 10,11). The "26S proteasome" is composed of two general sub-structures: the catalytically active 20S core particle (CP), as well as a $19 \mathrm{~S}$ regulatory particle (RP) involved in substrate recognition, unfolding and translocation into the inner core. The $\mathrm{CP}$ is assembled of 14 structurally related alpha ( $\alpha 1-\alpha 7)$ and beta ( $\beta 1-$ $\beta 7)$ subunits, which associate as four stacked hetero-heptameric rings. The outer a-rings provide an entry gate to the inner proteolytic chamber composed of $\beta$-rings. Of the seven different beta subunits, three have been shown to have distinct catalytic activities as threonine proteases, cleaving peptides after hydrophobic ("chymotrypsin-like activity"), basic ("trypsin-like activity") or acidic ("peptidylglutamyl-hydrolysing activity") residues (reviewed in 12). Proteasomes degrade proteins in a highly processive fashion, releasing peptides with a median length of five residues (13). All cleavage sites are sequestered on the inside of the $\mathrm{CP}$, and access is controlled by the RP attached to the alpha rings on one or both ends (reviewed in 14). The RP is made up of 19 subunits, which form two subcomplexes referred to as lid and base. Six AAA+ (ㅁTPase asssociated with various activities) ATPases that assemble into a hexameric ring at the base of the RP function as the driving force in substrate unfolding and translocation. Other non-ATPase subunits are involved in various aspects of substrate recognition and processing.

Prokaryotic proteasomes were first discovered in the thermoacidophilic archaeon Thermoplasma acidophilus, where electron microscopy revealed the existence of proteolytically active particles similar in shape and size to eukaryotic proteasomes (15). In 
comparison to the eukaryotic $20 \mathrm{~S} \mathrm{CP}$, the subunit composition of the archaeal proteasome is less complex, which facilitated the determination of the first crystal structure of a proteasome from any of the three domains of life (16). The first bacterial proteasome was identified in the actinomycete Rhodococcus erythropolis (17). Later, 20S proteasomes were biochemically or genetically characterized in other members of this order, namely Mycobacterium smegmatis (18), Streptomyces coelicolor (19) and Frankia (20). Additionally, sequence information suggested the presence of proteasomes in Mtb (9) and Mycobacterium leprae (reviewed in 21). The notion that bacterial proteasomes are limited to actinomycetes was challenged when the Banfield group discovered peptides encoded by proteasomal genes in biofilm samples of the uncultivated, Gram-negative bacterium Leptospirillum using a mass-spectrometry-based approach (22). Thus far, actinomycetes and Leptospirillum remain the only known bacterial lineages with a proteasome system. It is hypothesized that both have independently acquired the gene clusters encoding this complex protease via lateral gene transfer events $(23,24)$.

Overall, the architecture of proteasomes from all three domains of life is remarkably similar: cylinder-shaped particles $15 \mathrm{~nm}$ in height and $11 \mathrm{~nm}$ in diameter are assembled in the $\alpha_{7} \beta_{7} \beta_{7} \alpha_{7}$ fashion. While cognate ATPases have been identified and genetically linked to 20S CPs in both archaea and bacteria, robust physical interactions have not been detected, suggestive of other factors required for proteolysis (25). In contrast, the eukaryotic $26 \mathrm{~S}$ holocomplex can be purified. Most prokaryotic CPs consist of only one a (PrcA) and one $\beta$ (PrcB) subunit, both of which form homoheptameric rings. This arrangement generally limits prokaryotic proteasomes to chymotryptic activity. The $M t b$ proteasome, which shares modest sequence similarity with the Thermoplasma $\mathrm{CP}$ (32\% for both $\alpha$ - and $\beta$-subunits) and a high degree of identity (65\%) with the Rhodococcus proteasome, is compliant with these features. However, several observations distinguish the $M t b$ proteasome from other prokaryotic 20S CPs. All $\beta$-subunits are synthesized with $\mathrm{N}$-terminal pro-peptides, which undergo autocatalytic processing to reveal the catalytic threonine residue. Unlike the Rhodococcus pro-peptide, which acts as an assembly-promoting factor, the $M t b$ pro-peptides represent a thermodynamic barrier to this process. Additionally, the $M t b$ proteasome shows broad substrate specificity despite the presence of a single type of catalytic $\beta$-chain. Topological features of the substrate-binding pocket that mimic that of corresponding surfaces in Saccharomyces cause this unusual characteristic $(26,27)$. It is notable that although proteasomes share certain properties with the four classes of energy-dependent proteases commonly found in bacteria, they are structurally and enzymatically distinct from ClpXP, HslUV, FtsH and Lon (reviewed in 28).

\section{Pupylation}

Almost all proteins targeted for proteasomal degradation in eukaryotes are tagged with ubiquitin (Ub). Ub is a small protein of 76 amino acids that can be covalently linked to other proteins via its $\mathrm{C}$-terminal di-glycine motif. Produced as an inactive precursor, $\mathrm{Ub}$ is proteolytically processed by deubiquitylases (DUBs) to expose the site of substrate attachment. "Ubiquitylation" (also referred to as ubiquitination) then proceeds in a multistep cascade (Figure 1). Initially, $\mathrm{Ub}$ is activated through adenylation at the $\mathrm{C}$-terminus. A high-energy thioester bond to the active site cysteine of a Ub activating enzyme ("E1") 
quickly replaces the mixed anhydride bond. Subsequently, Ub is transferred to the catalytic cysteine of a Ub conjugating enzyme ("E2"). Ub can then be transferred to its substrates by a Ub ligase ("E3"), resulting in the formation of an isopeptide bond between the C-terminal glycine of $\mathrm{Ub}$ and the $\varepsilon$-amino group of substrate lysines (reviewed in 29, 30). Substrate specificity is largely determined by the presence of more than $600 \mathrm{E} 3$ ligases (31) that are

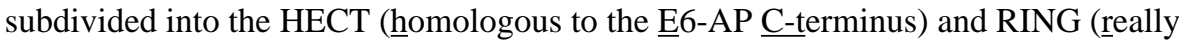
interesting new gene) classes (reviewed in 32,33$)$. Ub has seven lysine $(\mathrm{K})$ residues, which allows for the generation of branched poly-ubiquitin chains. Classically, tetra-Ub chains linked at K48 are regarded as the minimal signal for degradation (34), although an increasing number of studies report monoubiquitylation to be sufficient for recognition (35). Polyubiquitylated substrates can be recognized by specific Ub receptors located at the proteasome. Before substrates are fed into the $\mathrm{CP}$, proteasome-associated DUBs can cleave and recycle the poly-Ub chain (reviewed in 11).

Although there are striking structural and biochemical similarities between eukaryotic and prokaryotic CPs, it was unclear how proteins are targeted for proteasomal degradation in bacteria, which do not have Ub. Unsuccessful attempts to reconstitute the ATPasedependent degradation of proteasomal substrates in vitro suggested the involvement of additional cofactors. An important first step was the identification of endogenous mycobacterial proteasome substrates (36). Two proteins, malonyl CoA-acyl carrier protein transacylase (FabD) and ketopantoate hydroxymethyltransferase (PanB), accumulate in an $m p a$ null strain or in wild type $M t b$ treated with the mammalian proteasome inhibitor epoxomicin. Transcriptional analysis showed no difference in $f a b D$ or panB transcript levels, suggesting that increased protein abundance is not caused by induced gene expression. To further test these results, epitope-tagged $f a b D$ and $p a n B$ were placed under the control of a heterologous M. bovis hsp60 promoter and an Escherichia coli (E. coli) ribosome binding site to control for changes in transcription and translation initiation. Heterologously produced FabD and PanB accumulate in mpa and pafA mutant strains, as well as in wild type $M t b$ treated with proteasome inhibitor (36), thus further strengthening the functional association between Mpa, PafA and the proteasome. Interestingly, the same study also demonstrated that Mpa levels are regulated by the concerted efforts of Mpa, PafA and the proteasome. Mpa accumulates in strains producing mutant Mpa lacking its ATPase activity (37), as well as in the pafA null strain or in wild type $M t b$ after chemical inhibition of the proteasome (36). Thus, Mpa is autoregulated via proteasomal degradation.

In order to determine additional components of the Mtb proteasome system, Darwin and coworkers performed a genetic screen for interaction partners of Mpa, utilizing an E. coli twohybrid system (38). Screening an Mtb genomic library of $\sim 100,000$ fragments for positive interactions with an Mpa bait fusion, the authors identified Rv2111c, which is the gene located immediately upstream of $\operatorname{prcBA}$ and predicted to form an operon with the $\mathrm{CP}$ genes. However, addition of Rv2111c to CP and Mpa did not stimulate degradation of FabD in vitro, suggesting the requirement of additional factors for proteolysis. The authors hypothesized that mycobacteria-specific co-factors were required to promote proteolysis. Consequently, they employed a mycobacterial two-hybrid system (39), which enables the study of protein-protein associations in Mycobacterium smegmatis (Msm). Surprisingly, 
Rv2111c and a proteasome substrate (FabD) showed a strong interaction in this system, which was further tested by co-purification of the recombinant proteins from Msm. Unexpectedly, these experiments yielded a stable covalent complex between the two proteins. Mass spectrometry revealed the formation of an isopeptide bond between the Cterminal residue of Rv2111c and a particular FabD lysine residue (K173). Contrary to ubiquitin, Rv2111c does not encode a C-terminal di-glycine motif, but instead contains this motif at the penultimate position, followed by glutamine. Moreover, the C-terminal residue is deamidated to glutamate prior to conjugation. Mutation of the pupylated lysine of FabD (K173A) results in stabilization of the protein in Msm, indicating that modification of proteins with Rv2111c is a signal for degradation. Based on its functional, albeit not biochemical analogy to ubiquitin Rv2111c was named Pup. Interestingly, while numerous "pupylated" proteins can be observed by immunoblot in total lysates from wild type and mpa Mtb strains, no anti-Pup reactive bands are detectable in a pafA null strain, indicating the involvement of PafA in substrate conjugation (40).

In an independent study, Burns et al. showed that a homologue of Rv2111c (MSMEG_3896) acts as a protein modifier in Msm. Production of epitope-tagged Pup in $M s m$ was sufficient to modify numerous proteins, demonstrating that the signal for proteasomal degradation is conserved in both pathogenic and non-pathogenic mycobacteria (41). Taken together, these studies showed for the first time that some bacteria are able to utilize a posttranslational tagging system reminiscent of ubiquitylation in eukaryotes, thereby marking a turning point in the understanding of prokaryotic proteasome biology.

Significant progress has been made in elucidating the biochemistry of this post-translational modifier (Figure 2). Pup is an intrinsically disordered protein of 64 amino acids in length (42-44), which is in stark contrast to Ub and other related eukaryotic modifiers that have a conserved $\beta$-grasp fold (reviewed in 45). Intrinsically disordered proteins lack a compact structure under physiological conditions, although binding-induced folding of the C-terminal half of Pup aids its recognition by Mpa (see below).

At the time of its discovery, pafA did not share any homology with proteins of known function $(7,46)$. Later, sensitive sequence analysis classified PafA as a member of the carboxylate-amine/ammonia ligase superfamily. Proteins of this class catalyze a two-step ligase reaction that results in an amide linkage between an amino and a carboxylate group. Aravind and colleagues proposed a model in which PafA phosphorylates the $\gamma$-carboxylate of the C-terminal glutamate of Pup ( Pup $_{\mathrm{Glu}}$ ), priming it for nucleophilic attack by the $\varepsilon$-side chain amino group of a target lysine, which results in formation of an isopeptide bond (47).

In vitro, PafA is sufficient to conjugate Pup $_{\mathrm{Glu}}$ to mycobacterial proteasome substrates in an ATP-dependent fashion (48). Site-directed mutagenesis of any residues predicted to coordinate $\mathrm{Mg}^{2+}$ or ATP abrogated pupylation in vivo, supporting the predicted model (49). A report by Weber-Ban and co-workers tested the Aravind lab's hypothesis and found that PafA indeed catalyzes ligation of Pup to substrates via a two-step reaction mechanism. The authors were able to detect a phosphorylated Pup intermediate bound to PafA following ATP hydrolysis (50). Nuclear magnetic resonance (NMR) spectroscopy revealed that coupling to substrates occurs indeed via the side chain carboxylate of Pup, ruling out any possible involvement of the a-carboxylate of its C-terminus (51). 
Purification of epitope-tagged Pup from Msm yields both Pup $\mathrm{Glu}_{\text {alu }}$ and Pup $\mathrm{P}_{\mathrm{Gln}}$. The

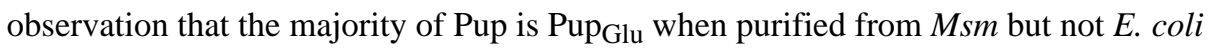
suggested that an enzymatic activity is responsible for this conversion (40). Aravind and colleagues noticed that the $M t b$ genome encodes two paralogues of PafA and thus proposed for the ligase to assemble into a heterodimer (47). This hypothesis was refuted in a series of experiments that demonstrated the paralogue of PafA has a distinct function: Dop (deamidase of Pup) converts the C-terminal glutamine residue of Pup to glutamate. Unlike PafA, Dop appeared to utilize ATP only as a cofactor, as no hydrolysis and accompanying release of ADP or AMP was detected. Consolidating this finding, deamidation also proceeds in presence of a non-hydrolyzable nucleotide analogue, such as ATP $\gamma \mathrm{S}$, albeit at a lower rate (48).

Sequential action of Dop and PafA constitutes a two-step pupylation pathway. However, in vitro, the requirement for Dop can be bypassed, as PafA is sufficient to conjugate Pup $\mathrm{P}_{\mathrm{Glu}}$ to target substrates. Actinomycetes encode pup either with a C-terminal glutamine residue, like mycobacteria, or with glutamate (e.g. Corynebacterium $s p$ ) thus eliminating the need for a deamidation step (reviewed in 52). This observation hinted to another function for Dop.

\section{Depupylation}

In humans, over 70 deubiquitylases (DUBs) are responsible for removing Ub chains from modified substrates. Their action prevents the turnover of $\mathrm{Ub}$ together with proteins targeted to the $26 \mathrm{~S}$ proteasome, so that $\mathrm{Ub}$ can be recycled for subsequent conjugation reactions. Under certain circumstances, removal of $\mathrm{Ub}$ rescues a protein from degradation (reviewed in $53)$.

It was hypothesized that mycobacterial cells have the ability to resolve the bond between Pup and its substrate. A series of experiments reported in two independent studies identified Dop, the deamidase of Pup, as a bifunctional enzyme with depupylase (DPUP) activity. Darwin and coworkers noted that numerous pupylated substrates (the "pupylome") disappeared over time in the presence of ATP but absence of proteasomes. In addition, specific recombinant pupylated proteasome substrates (Pup FabD and Pup Ino1 (myoinositol-1-phosphate synthase)) were "depupylated" in mycobacterial lysates. However, Mtb lysates of a dop mutant lack the ability to remove Pup from substrates. The authors reasoned that a putative depupylation reaction is chemically identical to the previously described deamidation reaction: both activities involve hydrolysis of an amide bond at the C-terminal residue of Pup. Indeed, purified Dop is able to depupylate different proteasomal substrates in vitro, releasing Pup ${ }_{G l u}$. As for deamidation, ATP binding appears to be critical for functionality of the enzyme (54). Similarly, Weber-Ban and co-workers demonstrated that Dop functions as a depupylase in Msm. The proteasome substrate Pup PanB is stable in lysates of an Msm dop mutant, while depupylation is restored in a complemented strain. Mass spectrometry analysis of the products released after incubation of Dop with Pup PanB identifies Pup with a C-terminal glutamate residue, demonstrating that the enzyme breaks the isopeptide bond between Pup and a substrate (55). Dop is a strict isopeptidase: unlike certain DUBs, which can cleave linear Ub chains, Dop does not show any reactivity against linear Pup-substrate fusions (54-56). Going forward, it will be critical to determine if 
depupylation of substrates can rescue them from degradation by the proteasome or if this step facilitates their delivery into the catalytic core of the protease.

Assessment of Dop's DPUP activity in vivo was complicated by its requirement for deamidation of Pup. In Msm, ectopic expression of $p p_{G l u}$ in a dop mutant is sufficient to restore pupylation to wild type levels (57). Interestingly, the pupylation defect of an Mtb dop

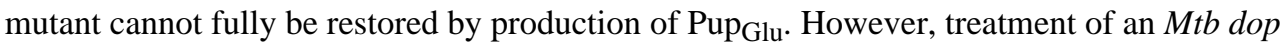
strain expressing pup $_{G l u}$ with the proteasome-inhibitor epoxomicin restores a robust pupylome. This indicates that the DPUP activity of Dop is essential to maintain steady-state levels of pupylation through the recycling of Pup (49).

Interestingly, Mpa contributes to depupylation. Over-production of Pup results in the rapid degradation of Ino1 in Msm. In an Msm mpa mutant, Pup Ino1 accumulates robustly but is virtually undetectable in wild type or proteasome deletion strains $(54,58)$. This suggested Mpa helps facilitate depupylation. Along these lines, Weber-Ban and colleagues demonstrated that Mpa enhances depupylation in vitro. Substrate unfolding by Mpa appears to increase the accessibility of the isopeptide bond for subsequent cleavage $(54,55)$. This result implies that Dop interacts with pupylated proteins subsequent to or concurrent with Mpa.

The crystal structures of Dop and PafA homologues from Acidothermus cellulolyticus and Corynebacterium glutamicum, respectively, have been solved (59). Notwithstanding moderate conservation on a primary sequence level (38\% sequence identity), the two proteins show high structural similarity. It appears that PafA and Dop arose from a gene duplication event that results in the production of enzymes with opposing activities. One might assume that the shared structural elements of PafA and Dop were conserved to accommodate Pup binding. As previously predicted, the large N-terminal domain of both PafA and Dop adopts a carboxylate-amine ligase fold. A smaller C-terminal domain is unique to these proteins. Even though the active site of both enzymes is located in a particular structural element (referred to as $\beta$-sheet cradle) present in the $\mathrm{N}$-terminal domain, mutation analysis has demonstrated that the C-terminus of PafA and Dop are critical for function $(49,59,60)$.

While multiple differences in the molecular architecture of the enzymes have been identified, site-directed mutagenesis or structural analysis did not identify a singular determinant of Dop activity (59). In another study, Burns et al. utilized an electrophilic trap consisting of Pup modified with a C-terminal glutamine mimic to identify a nucleophilic residue in Dop. Labeling of aspartate 95, an atypical nucleophile for a protease, classified Dop as an unusual amidase whereby the deamidation or depupylation reaction proceeds via an anhydride intermediate (61). Although this model is supported by the crystal structure, further experimentation is necessary to definitively prove this unusual protease activity.

\section{Genomic organization of the Pup-proteasome locus}

All PPS-associated genes identified to date are localized in direct proximity to the core proteasome (Figure 3). In mycobacteria, pup is encoded immediately upstream of prcBA and the overlap of the stop and start codons of pup and $\operatorname{prcB}$, as well as $\operatorname{prcB}$ and $\operatorname{prc} A$ suggest 
translational coupling. Usually, dop precedes this operon, while pafA is encoded further downstream, separated by several presumably unrelated genes. The mpa gene is found several open reading frames upstream of $d o p$.

It is notable that Corynebacteria encode homologues of all genes associated with (de)pupylation, despite lacking the proteasomal core genes (reviewed in 62). The presence of mpa in these organisms is particularly intriguing and may suggest that pupylation can serve as a degradation tag in absence of the proteasome. It is tempting to speculate that in these organisms, Mpa interacts with other degradation machines to catalyze turnover of pupylated substrates.

Perhaps somewhat surprisingly, little to nothing is known about the expression of proteasome-associated genes. Future studies will need to more closely examine if the PPS is differentially regulated under certain conditions.

\section{Substrate recognition by the mycobacterial proteasome}

The regulatory particle of the eukaryotic proteasome contains two subunits designated as intrinsic ubiquitin receptors (63). Although a similar arrangement is missing from the $M t b$ 20 S proteasome, Mpa interacts strongly with Pup (40). Optimal degradation of substrates by the $26 \mathrm{~S}$ proteasome requires both a proteasome binding signal (e.g. modification with $\mathrm{Ub}$ ) and an unstructured region to initiate degradation (reviewed in 14). NMR studies determined that the C-terminal half of Pup interacts with Mpa $(42,44)$. Co-crystallization experiments of Mpa and Pup further elucidated the mechanistic details of recognition: Pup binds the $\mathrm{N}$ terminal coiled coil domains of an Mpa hexamer through hydrophobic interactions. This contact induces the formation of an a-helix in the central part of Pup (64). Burns et al. demonstrated that this region of Pup also facilitates interaction with enzymes involved in pupylation, while the $\mathrm{N}$-terminal half is required to initiate unfolding and degradation. Thus, Pup itself provides the two components of the degron necessary for proteasomal degradation (58).

\section{Lack of a pupylation motif}

Several proteomics studies identified targets of pupylation in Mtb and Msm. Purification of proteins conjugated to Pup coupled with mass spectrometry identified $604 \mathrm{Mtb}$ proteins, representing $\sim 15 \%$ of the predicted proteome. A Pup attachment site was identified for 55 of these proteins (65). Two separate Msm pupylome analyses identified 52 (66) and 41 proteins (67), respectively, with confirmed pupylation sites. In all cases Pup was attached to substrates via a lysine residue, corroborating initial observations. Furthermore, Pup does not appear to form polymeric chains as seen in ubiquitylation. Determining the functional assignment of proteins identified in the Mtb pupylome, three categories appeared to be overrepresented when compared to their presence in the genome: intermediary metabolism, lipid metabolism and detoxification/virulence (65). This functional clustering of Pup targets is preserved in Msm (67). Interestingly, the authors of one study identified significant overlap between the Msm pupylome and the Mtb nitrosoproteome, suggesting that the PPS could have an important role in the turnover of damaged proteins in response to RNI challenge (66). 
The pupylome studies did not reveal a consensus primary sequence recognition motif at the site of modification. Generally, the attachment sites for posttranslational protein modifiers are too diverse to define a consensus sequence. SUMO (small ubiquitin-related modifier) is the only notable exception, as many of its target proteins contain an acceptor lysine within a $\psi \mathrm{KxD} / \mathrm{E}$ motif, where $\psi$ is a large, hydrophobic residue (reviewed in 68). Although Dorrestein and colleagues mapped the pupylation sites on available crystal structures to the periphery of the target subunits (66), it remains mysterious how a single ligase PafA can act on potentially all pupylation substrates and discriminate them from non-targets in the cell.

Darwin and coworkers reconstituted the pupylation pathway in E. coli, a surrogate host that does not natively encode the Pup machinery. Co-expression of pafA and $p_{\text {up }}$ Glu is sufficient to pupylate mycobacterial substrates in E. coli. Surprisingly, anti-Pup immunoblots revealed that numerous $E$. coli proteins were also modified in this system, suggesting that no additional mycobacteria-specific cofactors are required in the process of pupylation. The $E$. coli pupylome consists of 51 proteins with identified Pup attachment sites. The E. coli substrate phosphoenolpyruvate protein phosphotransferase I (PtsI), which does not have a homolog in mycobacteria, could be pupylated in $M s m$, demonstrating that a completely foreign protein can be recognized by a native pupylation system. However, pupylation is not an arbitrary event, as not every protein can be pupylated. For example, dihydrolipoamide acyltransferase (DlaT) is pupylated neither in mycobacteria, nor in the E. coli system, despite having 29 lysines. Furthermore, there is a distinct preference for the modified lysine residue in mycobacteria, which is particularly striking in $\mathrm{FabD}$, where all eight lysines are surface-exposed but only one is targeted for pupylation in the native system. The authors speculate that the unmodified lysines are shielded from PafA by other components of the multi-enzyme fatty acid synthesis pathway in which FabD participates (60). It remains to be determined how protein-protein interactions shape pupylation in general, as the current knowledge cannot rule out involvement of additional factors in target selection or delivery of substrates to the proteasome.

\section{Characterization of Pup-proteasome mutants}

$M t b$ mutants in $m p a$ and pafA are highly attenuated in a mouse model of infection as measured by reduced bacterial load, gross pathology and histologically alleviated pneumonitis after a low dose aerosol challenge ( 200 bacilli/mouse) (7). Time-to-death experiments showed that contrary to mice infected with wild type $M t b$, which show a median survival of $\sim 1.5$ years after infection, mice infected with mpa or pafA remained alive and healthy, while maintaining robust body weight and exhibiting no symptoms beyond normal aging $(36,37)$. As explained earlier, Nathan and co-workers observed increased in vitro sensitivity to acidified nitrite for $m p a$ and $p a f A$ mutants, raising the question if higher susceptibility to RNI in vivo was the reason for their attenuation in mouse infections. In mammals, NO is produced by three isoforms of nitric oxide synthase: endothelial (eNOS), neuronal (nNOS) or inducible/immune (iNOS) (reviewed in 69). Phagocytes are prolific generators of NO, contributing to the control of various infections, such as Leishmania major and Coxsackie B3 virus (reviewed in 2). Mice genetically inactivated for iNOS $\left(\mathrm{iNOS}^{-/-}\right.$) or treated with a chemical inhibitor of iNOS are extremely susceptible to $M t b$ infection compared to wild type mice (1). Low dose aerosol infection of iNOS ${ }^{-/}$mice with 
wild type $M t b$ results in death after two to three months $(7,36,37)$. In contrast, iNOS ${ }^{-1-}$ mice infected with mpa or pafA mutant strains live significantly longer than $\mathrm{iNOS}^{-/-}$mice infected with wild type $M t b$, but nonetheless eventually all die $(36,37)$. Hence, genetic inactivation of iNOS partially restores susceptibility to mpa and pafA mutants, indicating the importance of these genes to resist NO.

In another study, Bishai and colleagues screened a library of transposon mutants in the CDC1551 background for mutants displaying morphological changes on agar media. Three independent mutants of mpa (MT2175) consistently formed smaller colonies than wild type $\mathrm{Mtb}$ on solid media. To facilitate a more in-depth analysis of the consequences caused by absence of this gene, the authors generated a deletion disruption mutant, in which a hygromycin resistance cassette replaced more than $90 \%$ of the coding sequence of $m p a$. Growth of the $\Delta m p a:: h y g$ mutant in rich medium (7H9) was impaired: the average in vitro doubling time was determined to be $22 \mathrm{~h}$, compared to $18 \mathrm{~h}$ for wild type CDC1551. High dose aerosol infection of BALB/c mice shows an infection profile similar to that obtained with the H37Rv mpa mutant. The authors also observed significantly less pathology in mice infected with the $\Delta m p a:: h y g$ mutant, characterized by reduced levels of granuloma-like immune infiltrates in the lungs and no changes in body weight. Furthermore, mice infected with the $\Delta m p a::$ hyg mutant did not succumb to infection until the experiment was terminated at 180 days, while the median survival time for wild type $M t b$ infected mice was 25 days (70).

Disruption of dop also sensitizes Mtb to RNI in vitro and severely attenuates survival and growth in mice. The degree of attenuation, as assessed by histopathology and bacterial load, is similar between dop, mpa and pafA mutants, supporting the hypothesis that a common pathway functionally links all three genes $(7,49)$. Mice infected with any of these mutants do not show severe tissue destruction and infiltration of lymphocytes characteristic of wild type $M t b$. Bishai and co-workers dubbed their $\Delta m p a:$ : hyg strain as an "immunopathology mutant" due to its ability to persist in the lungs without causing significant immune-induced disease. Interferon gamma production is not induced in mice infected with a $\Delta m p a:$ :hyg mutant compared to wild type $M t b$ at three weeks post infection. Interestingly, their $\Delta$ mpa::hyg strain offered some immunological protection against virulent $M t b$ challenge in mice (70).

The reduced pathology caused by infections with PPS mutants is likely a direct result of a 100-1000 fold lower bacterial burden during the persistent stage of the infection; however, we cannot exclude the possibility that it is the dysregulation of a specific protein or subset of proteins in proteasomal degradation-deficient mutants that causes the changes in pathology.

The genetic screen for mutants hypersensitive to NO did not yield any mutants with insertions in the core proteasomal genes $\operatorname{prcBA}$. Employing transposon in situ hybridization (TraSH) of a saturating mutant library, Rubin and co-workers identified $\operatorname{prcBA}$ but not $m p a$ or pafA among a set of genes required for cellular growth in vitro (71), suggesting $\operatorname{prcBA}$ are essential. A conditional gene silencing system using a tetracycline-responsive promoter facilitated studies to test the essentiality of $\operatorname{prcBA}$ (72). This study indeed showed that $\operatorname{prcBA}$ is required for normal growth in vitro. Later, a deletion-disruption strain in which $\operatorname{prcBA}$ 
was replaced with a hygromycin resistance cassette ( $\triangle p r c B A:: h y g)$ was characterized (73). Although the mutant is viable, it grows very slowly. Not surprisingly, infection with either of the $\operatorname{prcBA}$ mutants results in fewer bacilli in the lungs of C57BL/6 mice compared to infection with wild type $M t b$. Importantly, the number of bacteria decreased dramatically in later weeks, suggesting that the proteasome core protease is essential for mycobacterial persistence in mice $(72,73)$.

A pup mutant has never been reported. Like $\operatorname{prcBA}$, it is predicted to be essential based on TraSH (71). Because pup is predicted to be co-transcribed and translated with $\operatorname{prcBA}$, disruption of pup would likely result in polar effects on $\operatorname{prcBA}$ expression. Pup is undetectable in either a dop or pafA mutant, presumably because unconjugated Pup is highly unstable $(40,49)$. Thus, one would presume that these mutants would be phenotypically similar if not identical to a hypothetical pup mutant.

It is striking that $\operatorname{prcBA}$ mutants display an in vitro growth defect absent from mpa, pafA and dop null strains $(7,49)$. To discern if the mycobacterial proteasome has functionality beyond its proteolytic activity, Ehrt and colleagues mutated the catalytic threonine residue of $\operatorname{PrcB}$ to alanine (T1A). The active-site mutant assembles into a 20S complex with PrcA but production of this recombinant proteasome in a $\triangle p r c B A$ mutant does not rescue proteasomal activity. Surprisingly, production of this inactive proteasome complex is sufficient to complement the hypersusceptibility to NO. In contrast, proteasomal proteolysis is essential for persistence in mice (73). It is unclear how proteasomal activity is connected to certain phenotypes, but not others. It is possible that an inactive $20 \mathrm{~S}$ core structure could act as a "trap" to contain proteins that would normally be degraded. Trapping could result in (partial) inactivation of these proteins and may thus explain how an active site mutant of the Mtb 20S can still protect against certain stresses but not others.

It has been hypothesized that the mechanism of protection by the proteasome against NO damage involves the degradation of irreversibly oxidized or nitrated proteins to prevent toxicity to the bacterium. However, mpa, pafA and $p r c B A$-deficient strains are more resistant than wild type $M t b$ to hydrogen peroxide $(7,72)$. Although this could be due to compensatory induction of additional antioxidant pathways, these data suggest that mere turnover of accumulating damaged proteins does not explain the hypersensitivity of PPS mutants to NO.

The role of the $\mathrm{CP}$ in virulent mycobacteria appears to be distinct from that in nonpathogenic $M s m$, where disruption of the proteasome does not result in any discernible phenotype (18) (Table 1). Interestingly, unlike $M t b, M s m$ encodes a homologue of the compartmentalized serine protease Lon, which is the primary protease responsible for protein quality control in $E$. coli (reviewed in 74, 75). Hence, it is possible that production of Lon compensates for the effects caused by deletion of the proteasome in Msm, however, no current experimental evidence tests this assumption.

\section{Role of the proteasome in transcriptional regulation}

A microarray study was conducted to investigate a potential role of the proteasome in transcriptional regulation. Self-compartmentalizing proteases in both prokaryotes and 
eukaryotes can directly or indirectly regulate stability of transcription factors (reviewed in $74,76)$. Comparison of the early stationary phase transcriptomes from wild type $M t b$ to $m p a$ and pafA mutants revealed that a shared set of genes [less than $2 \%$ of all predicted open reading frames (ORFs)] is differentially regulated in the strains deficient in proteasomemediated degradation, suggesting that Mpa and PafA do not have independent roles affecting gene expression.

Among the genes upregulated in the mpa and pafA mutants are members of the Zur (zinc uptake regulator) regulon. Zur acts as a zinc-sensing transcriptional repressor that directly binds to a conserved palindromic regulatory element found in eight $M t b$ promoters. Under zinc-limiting conditions, Zur is released from its operators and gene expression is induced (77). Among the Zur-regulated genes identified in the microarray was the esx-3 (esat-6, region 3) operon. The Esx-3 cluster, which is required for optimal growth of Mtb in vitro, encodes a type VII secretion system involved in iron and zinc uptake $(78,79)$. Under normal growth conditions, the levels of free zinc in the cytosol are almost negligible, as zinccoordinating enzymes and ribosomes largely sequester zinc. Several ribosomal genes are controlled by the Zur regulon in $M t b$. These genes encode paralogs of zinc-binding proteins, which lack the metal-binding motif (77). In Bacillus subtilis, zinc-binding ribosomal proteins are replaced under zinc-limiting conditions, allowing for mobilization of stored zinc, as well as de novo synthesis of ribosomes $(80,81)$. Transcript levels of zur itself are unchanged in mpa and pafA deficient mycobacteria and it is unclear how absence of proteasomal degradation results in the deregulation of this regulon. Maintaining cellular metal homeostasis is critical to sustain key metabolic processes without exposing cells to the toxic effects exhibited by excess zinc or free iron (reviewed in 82). Hence, altered intracellular zinc levels throughout the course of infection could contribute to the attenuation of mpa and pafA mutants in vivo.

Microarray analysis further identified a novel copper-responsive regulon, expression of which is downregulated in the mpa and pafA mutants. A common repressor RicR (regulated in copper repressor) binds to a palindromic sequence (5'-TACCC-N $5_{5}-\mathrm{G} /$ AGGTA) located between the $-10 /-35$ sites in the promoter of several physically unlinked genes. In the presence of copper, RicR dissociates from promoters resulting in transcriptional derepression of ricR itself, mymT (a copper metallothionein), $\operatorname{lpq} S$ (a putative lipoprotein), Rv2963 (a putative permease) and an operon (socAB, small $\underline{\mathrm{O} F}$ induced by copper A and B) of unknown function. A $\Phi$ MycoMarT7 transposon mutant of ricR in the CDC1551 background grows slower than wild type $M t b$ and never reaches the same culture density under routine culture conditions. Perhaps not surprisingly, a ricR mutant is hyper-resistant to copper in vitro, most likely because one or more of the gene products of the regulon combat toxicity associated with excess levels of this transition metal. For example, MymT is robustly produced in a ricR null mutant (83) and binds up to six reduced copper ions to protect $M t b$ from copper-mediated toxicity (84).

It is noteworthy that several of the genes underlying regulation by RicR (lpqS, mymT and $\operatorname{soc} A B$ ) are only found in pathogenic mycobacteria, suggesting that their function is critical to adapt to the specific niche inside the animal host. Interestingly, non-proteasomal ATPdependent proteases have been implicated in the regulation of virulence gene expression in 
other bacteria (reviewed in 28). This study, as well as others that recently reported the identification of copper-responsive regulators in different pathogens, contribute to an emerging notion of copper as an important antimicrobial defense mechanism exhibited by macrophages (reviewed in 85).

The mechanism for deregulation of the RicR and Zur regulons in proteasome-mediated degradation deficient mutants of $M t b$ is unknown at this point. However, it is interesting that the transcriptome of these strains appears to be responding to low metal conditions, possibly as a result of accumulation of one or more metal-binding proteins in degradation deficient strains. In response to inflammation, plasma nutrient availability is altered to restrict the growth of pathogenic invaders: as a result of the acute phase response, zinc levels are decreased, while the amount of copper in the plasma increases (reviewed in 86). Hence, adaptation to the particular environment inside the host to achieve metal homeostasis is critical during infection.

\section{Outlook and remaining questions}

The discovery of the PPS in Mtb commenced a period of exciting findings in mycobacteria. Posttranslational protein modifiers, previously thought limited to eukaryotes, have since been shown to exist in a similar fashion in archaea, where conjugation of two ubiquitin-like proteins termed SAMP1/SAMP2 (ㅍmall archaeal modifier proteins) presumably targets proteins for proteasomal degradation (87). While much progress can be denoted on elucidating the biochemistry of pupylation, some major questions remain and others emerged.

For one, the regulation of pupylation is poorly understood. While a myriad of enzymes is devoted to target selection in the eukaryotic ubiquitylation pathway, one enzyme appears to fulfill this role in mycobacteria. Future studies will have to be directed at understanding whether or not additional cellular factors aid substrate recognition, as no structural or primary sequence motif unites all pupylation targets. Elucidating the principles that regulate modification with Pup might also shed light as to whether pupylation has non-proteasomal functions. Several pupylated proteins do not accumulate in $M t b$ strains deficient in proteasome-mediated degradation (65) and a recent report suggests that pupylation can inactivate Mpa function in vitro (88). It remains elusive at this time whether or not this finding has physiological relevance and how a degradation-independent regulatory function of pupylation could be distinguished in a cellular context, as chain formation or differential linkage of Pup to substrates have yet to be observed.

The presence of a proteasome provides a fitness advantage to mycobacteria during infection. However, the link between proteasome function and pathogenesis has not yet been fully elucidated. Is the accumulation of an individual substrate toxic to the cell, resulting in the observed NO hypersensitivity? Or is the large overlap between nitrosoproteome and pupylome indicative of the proteasome's role to relieve multifactorial insults in response to stress? Although the mechanism of how the mycobacterial proteasome affects transcription is not understood, it is remarkable that it appears connected to metal homeostasis. Reaction of NO with iron-sulfur clusters has been shown to destabilize the transition metal center. 
Release of free iron can promote the production of hydroxyl radicals through Fenton chemistry (reviewed in 3,4). Additionally, NO can displace copper from Mtb proteins (84). Hence, it is tempting to speculate that a combination of proteolysis-dependent and independent actions of the proteasome influences the bacterial transcriptional response to oxidative and nitrosative stress, thereby relieving some of the toxicity caused by NO.

Completing the characterization of the PPS will undoubtedly broaden our understanding of $M t b$ biology. The importance of this pathway for virulence makes the pupylation machinery an attractive drug target. One of the exciting new endeavors of the young field of bacterial proteasome biology will be the design of chemotherapeutics against one of the world's most successful pathogens.

\section{Acknowledgments}

Pup-proteasome research in the Darwin lab is supported by NIH grants AI088075 and HL92774, and the Irma T. Hirschl Trust. K.H.D. holds an Investigators in the Pathogenesis of Infectious Disease Award from the Burroughs Wellcome Fund.

\section{References}

1. MacMicking JD, North RJ, LaCourse R, Mudgett JS, Shah SK, Nathan CF. Identification of nitric oxide synthase as a protective locus against tuberculosis. Proc Natl Acad Sci U S A. 1997; 94:52438. [PubMed: 9144222]

2. Nathan C, Shiloh MU. Reactive oxygen and nitrogen intermediates in the relationship between mammalian hosts and microbial pathogens. Proc Natl Acad Sci U S A. 2000; 97:8841-8. [PubMed: 10922044]

3. Alvarez B, Radi R. Peroxynitrite reactivity with amino acids and proteins. Amino Acids. 2003; 25:295-311. [PubMed: 14661092]

4. Fang FC. Antimicrobial reactive oxygen and nitrogen species: concepts and controversies. Nat Rev Microbiol. 2004; 2:820-32. [PubMed: 15378046]

5. Szabo C. Multiple pathways of peroxynitrite cytotoxicity. Toxicol Lett. 2003; 140-141:105-12.

6. Ernst JD. The immunological life cycle of tuberculosis. Nat Rev Immunol. 2012; 12:581-91. [PubMed: 22790178]

7. Darwin KH, Ehrt S, Gutierrez-Ramos JC, Weich N, Nathan CF. The proteasome of Mycobacterium tuberculosis is required for resistance to nitric oxide. Science. 2003; 302:1963-6. [PubMed: 14671303]

8. Wolf S, Nagy I, Lupas A, Pfeifer G, Cejka Z, Muller SA, Engel A, De Mot R, Baumeister W. Characterization of ARC, a divergent member of the AAA ATPase family from Rhodococcus erythropolis. J Mol Biol. 1998; 277:13-25. [PubMed: 9514743]

9. Cole ST, Brosch R, Parkhill J, Garnier T, Churcher C, Harris D, Gordon SV, Eiglmeier K, Gas S, Barry CE 3rd, Tekaia F, Badcock K, Basham D, Brown D, Chillingworth T, Connor R, Davies R, Devlin K, Feltwell T, Gentles S, Hamlin N, Holroyd S, Hornsby T, Jagels K, Krogh A, McLean J, Moule S, Murphy L, Oliver K, Osborne J, Quail MA, Rajandream MA, Rogers J, Rutter S, Seeger K, Skelton J, Squares R, Squares S, Sulston JE, Taylor K, Whitehead S, Barrell BG. Deciphering the biology of Mycobacterium tuberculosis from the complete genome sequence. Nature. 1998; 393:537-44. [PubMed: 9634230]

10. Murata S, Yashiroda H, Tanaka K. Molecular mechanisms of proteasome assembly. Nat Rev Mol Cell Biol. 2009; 10:104-15. [PubMed: 19165213]

11. Finley D. Recognition and processing of ubiquitin-protein conjugates by the proteasome. Annu Rev Biochem. 2009; 78:477-513. [PubMed: 19489727]

12. Rubin DM, Finley D. Proteolysis. The proteasome: a protein-degrading organelle? Curr Biol. 1995; 5:854-8. [PubMed: 7583140] 
13. Kisselev AF, Akopian TN, Woo KM, Goldberg AL. The sizes of peptides generated from protein by mammalian 26 and $20 \mathrm{~S}$ proteasomes. Implications for understanding the degradative mechanism and antigen presentation. J Biol Chem. 1999; 274:3363-71. [PubMed: 9920878]

14. Schrader EK, Harstad KG, Matouschek A. Targeting proteins for degradation. Nat Chem Biol. 2009; 5:815-22. [PubMed: 19841631]

15. Dahlmann B, Kopp F, Kuehn L, Niedel B, Pfeifer G, Hegerl R, Baumeister W. The multicatalytic proteinase (prosome) is ubiquitous from eukaryotes to archaebacteria. FEBS Lett. 1989; 251:12531. [PubMed: 2502434]

16. Lowe J, Stock D, Jap B, Zwickl P, Baumeister W, Huber R. Crystal structure of the 20S proteasome from the archaeon T. acidophilum at 3.4 A resolution. Science. 1995; 268:533-9. [PubMed: 7725097]

17. Tamura T, Nagy I, Lupas A, Lottspeich F, Cejka Z, Schoofs G, Tanaka K, De Mot R, Baumeister W. The first characterization of a eubacterial proteasome: the 20S complex of Rhodococcus. Curr Biol. 1995; 5:766-74. [PubMed: 7583123]

18. Knipfer N, Shrader TE. Inactivation of the 20S proteasome in Mycobacterium smegmatis. Mol Microbiol. 1997; 25:375-83. [PubMed: 9282749]

19. Nagy I, Tamura T, Vanderleyden J, Baumeister W, De Mot R. The 20S proteasome of Streptomyces coelicolor. J Bacteriol. 1998; 180:5448-53. [PubMed: 9765579]

20. Pouch MN, Cournoyer B, Baumeister W. Characterization of the 20S proteasome from the actinomycete Frankia. Mol Microbiol. 2000; 35:368-77. [PubMed: 10652097]

21. Lupas A, Zuhl F, Tamura T, Wolf S, Nagy I, De Mot R, Baumeister W. Eubacterial proteasomes. Mol Biol Rep. 1997; 24:125-31. [PubMed: 9228293]

22. Ram RJ, Verberkmoes NC, Thelen MP, Tyson GW, Baker BJ, Blake RC 2nd, Shah M, Hettich RL, Banfield JF. Community proteomics of a natural microbial biofilm. Science. 2005; 308:1915-20. [PubMed: 15879173]

23. Gille C, Goede A, Schloetelburg C, Preissner R, Kloetzel PM, Gobel UB, Frommel C. A comprehensive view on proteasomal sequences: implications for the evolution of the proteasome. $\mathrm{J}$ Mol Biol. 2003; 326:1437-48. [PubMed: 12595256]

24. De Mot R. Actinomycete-like proteasomes in a Gram-negative bacterium. Trends Microbiol. 2007; 15:335-8. [PubMed: 17587582]

25. Wang T, Li H, Lin G, Tang C, Li D, Nathan C, Darwin KH, Li H. Structural insights on the Mycobacterium tuberculosis proteasomal ATPase Mpa. Structure. 2009; 17:1377-85. [PubMed: 19836337]

26. Hu G, Lin G, Wang M, Dick L, Xu RM, Nathan C, Li H. Structure of the Mycobacterium tuberculosis proteasome and mechanism of inhibition by a peptidyl boronate. Mol Microbiol. 2006; 59:1417-28. [PubMed: 16468986]

27. Lin G, Hu G, Tsu C, Kunes YZ, Li H, Dick L, Parsons T, Li P, Chen Z, Zwickl P, Weich N, Nathan C. Mycobacterium tuberculosis prcBA genes encode a gated proteasome with broad oligopeptide specificity. Mol Microbiol. 2006; 59:1405-16. [PubMed: 16468985]

28. Butler SM, Festa RA, Pearce MJ, Darwin KH. Self-compartmentalized bacterial proteases and pathogenesis. Mol Microbiol. 2006; 60:553-62. [PubMed: 16629660]

29. Husnjak K, Dikic I. Ubiquitin-binding proteins: decoders of ubiquitin-mediated cellular functions. Annu Rev Biochem. 2012; 81:291-322. [PubMed: 22482907]

30. Kerscher O, Felberbaum R, Hochstrasser M. Modification of proteins by ubiquitin and ubiquitinlike proteins. Annu Rev Cell Dev Biol. 2006; 22:159-80. [PubMed: 16753028]

31. Li W, Bengtson MH, Ulbrich A, Matsuda A, Reddy VA, Orth A, Chanda SK, Batalov S, Joazeiro CA. Genome-wide and functional annotation of human E3 ubiquitin ligases identifies MULAN, a mitochondrial E3 that regulates the organelle's dynamics and signaling. PLoS One. 2008; 3:e1487. [PubMed: 18213395]

32. Deshaies RJ, Joazeiro CA. RING domain E3 ubiquitin ligases. Annu Rev Biochem. 2009; 78:399_ 434. [PubMed: 19489725]

33. Rotin D, Kumar S. Physiological functions of the HECT family of ubiquitin ligases. Nat Rev Mol Cell Biol. 2009; 10:398-409. [PubMed: 19436320] 
34. Thrower JS, Hoffman L, Rechsteiner M, Pickart CM. Recognition of the polyubiquitin proteolytic signal. EMBO J. 2000; 19:94-102. [PubMed: 10619848]

35. Shabek N, Herman-Bachinsky Y, Buchsbaum S, Lewinson O, Haj-Yahya M, Hejjaoui M, Lashuel HA, Sommer T, Brik A, Ciechanover A. The size of the proteasomal substrate determines whether its degradation will be mediated by mono-or polyubiquitylation. Mol Cell. 2012; 48:87-97. [PubMed: 22902562]

36. Pearce MJ, Arora P, Festa RA, Butler-Wu SM, Gokhale RS, Darwin KH. Identification of substrates of the Mycobacterium tuberculosis proteasome. EMBO J. 2006; 25:5423-32. [PubMed: 17082771]

37. Darwin KH, Lin G, Chen Z, Li H, Nathan CF. Characterization of a Mycobacterium tuberculosis proteasomal ATPase homologue. Mol Microbiol. 2005; 55:561-71. [PubMed: 15659170]

38. Karimova G, Pidoux J, Ullmann A, Ladant D. A bacterial two-hybrid system based on a reconstituted signal transduction pathway. Proc Natl Acad Sci U S A. 1998; 95:5752-6. [PubMed: 9576956]

39. Singh A, Mai D, Kumar A, Steyn AJ. Dissecting virulence pathways of Mycobacterium tuberculosis through protein-protein association. Proc Natl Acad Sci U S A. 2006; 103:11346-51. [PubMed: 16844784]

40. Pearce MJ, Mintseris J, Ferreyra J, Gygi SP, Darwin KH. Ubiquitin-like protein involved in the proteasome pathway of Mycobacterium tuberculosis. Science. 2008; 322:1104-7. [PubMed: 18832610]

41. Burns KE, Liu WT, Boshoff HI, Dorrestein PC, Barry CE 3rd. Proteasomal protein degradation in Mycobacteria is dependent upon a prokaryotic ubiquitin-like protein. J Biol Chem. 2009; 284:3069-75. [PubMed: 19028679]

42. Chen X, Solomon WC, Kang Y, Cerda-Maira F, Darwin KH, Walters KJ. Prokaryotic ubiquitinlike protein pup is intrinsically disordered. J Mol Biol. 2009; 392:208-17. [PubMed: 19607839]

43. Liao S, Shang Q, Zhang X, Zhang J, Xu C, Tu X. Pup, a prokaryotic ubiquitin-like protein, is an intrinsically disordered protein. Biochem J. 2009; 422:207-15. [PubMed: 19580545]

44. Sutter M, Striebel F, Damberger FF, Allain FH, Weber-Ban E. A distinct structural region of the prokaryotic ubiquitin-like protein (Pup) is recognized by the N-terminal domain of the proteasomal ATPase Mpa. FEBS Lett. 2009; 583:3151-7. [PubMed: 19761766]

45. Hochstrasser M. Origin and function of ubiquitin-like proteins. Nature. 2009; 458:422-9. [PubMed: 19325621]

46. Festa RA, Pearce MJ, Darwin KH. Characterization of the proteasome accessory factor (paf) operon in Mycobacterium tuberculosis. J Bacteriol. 2007; 189:3044-50. [PubMed: 17277063]

47. Iyer LM, Burroughs AM, Aravind L. Unraveling the biochemistry and provenance of pupylation: a prokaryotic analog of ubiquitination. Biol Direct. 2008; 3:45. [PubMed: 18980670]

48. Striebel F, Imkamp F, Sutter M, Steiner M, Mamedov A, Weber-Ban E. Bacterial ubiquitin-like modifier Pup is deamidated and conjugated to substrates by distinct but homologous enzymes. Nat Struct Mol Biol. 2009; 16:647-51. [PubMed: 19448618]

49. Cerda-Maira FA, Pearce MJ, Fuortes M, Bishai WR, Hubbard SR, Darwin KH. Molecular analysis of the prokaryotic ubiquitin-like protein (Pup) conjugation pathway in Mycobacterium tuberculosis. Mol Microbiol. 2010; 77:1123-35. [PubMed: 20636328]

50. Guth E, Thommen M, Weber-Ban E. Mycobacterial ubiquitin-like protein ligase PafA follows a two-step reaction pathway with a phosphorylated pup intermediate. J Biol Chem. 2011; 286:44129. [PubMed: 21081505]

51. Sutter M, Damberger FF, Imkamp F, Allain FH, Weber-Ban E. Prokaryotic ubiquitin-like protein (Pup) is coupled to substrates via the side chain of its C-terminal glutamate. J Am Chem Soc. 2010; 132:5610-2. [PubMed: 20355727]

52. Burns KE, Darwin KH. Pupylation : A Signal for Proteasomal Degradation in Mycobacterium tuberculosis. Subcell Biochem. 2010; 54:149-57. [PubMed: 21222280]

53. Komander D, Clague MJ, Urbe S. Breaking the chains: structure and function of the deubiquitinases. Nat Rev Mol Cell Biol. 2009; 10:550-63. [PubMed: 19626045] 
54. Burns KE, Cerda-Maira FA, Wang T, Li H, Bishai WR, Darwin KH. "Depupylation" of prokaryotic ubiquitin-like protein from mycobacterial proteasome substrates. Mol Cell. 2010; 39:821-7. [PubMed: 20705495]

55. Imkamp F, Striebel F, Sutter M, Ozcelik D, Zimmermann N, Sander P, Weber-Ban E. Dop functions as a depupylase in the prokaryotic ubiquitin-like modification pathway. EMBO Rep. 2010; 11:791-7. [PubMed: 20798673]

56. Komander D, Reyes-Turcu F, Licchesi JD, Odenwaelder P, Wilkinson KD, Barford D. Molecular discrimination of structurally equivalent Lys 63-linked and linear polyubiquitin chains. EMBO Rep. 2009; 10:466-73. [PubMed: 19373254]

57. Imkamp F, Rosenberger T, Striebel F, Keller PM, Amstutz B, Sander P, Weber-Ban E. Deletion of dop in Mycobacterium smegmatis abolishes pupylation of protein substrates in vivo. Mol Microbiol. 2010; 75:744-54. [PubMed: 20025664]

58. Burns KE, Pearce MJ, Darwin KH. Prokaryotic ubiquitin-like protein provides a two-part degron to Mycobacterium proteasome substrates. J Bacteriol. 2010; 192:2933-5. [PubMed: 20233925]

59. Ozcelik D, Barandun J, Schmitz N, Sutter M, Guth E, Damberger FF, Allain FH, Ban N, WeberBan E. Structures of Pup ligase PafA and depupylase Dop from the prokaryotic ubiquitin-like modification pathway. Nat Commun. 2012; 3:1014. [PubMed: 22910360]

60. Cerda-Maira FA, McAllister F, Bode NJ, Burns KE, Gygi SP, Darwin KH. Reconstitution of the Mycobacterium tuberculosis pupylation pathway in Escherichia coli. EMBO Rep. 2011; 12:86370. [PubMed: 21738222]

61. Burns KE, McAllister FE, Schwerdtfeger C, Mintseris J, Cerda-Maira F, Noens EE, Wilmanns M, Hubbard SR, Melandri F, Ovaa H, Gygi SP, Darwin KH. Mycobacterium tuberculosis prokaryotic ubiquitin-like protein-deconjugating enzyme is an unusual aspartate amidase. J Biol Chem. 2012; 287:37522-9. [PubMed: 22942282]

62. Barandun J, Delley CL, Weber-Ban E. The pupylation pathway and its role in mycobacteria. BMC Biol. 2012; 10:95. [PubMed: 23198822]

63. Lander GC, Estrin E, Matyskiela ME, Bashore C, Nogales E, Martin A. Complete subunit architecture of the proteasome regulatory particle. Nature. 2012; 482:186-91. [PubMed: 22237024]

64. Wang T, Darwin KH, Li H. Binding-induced folding of prokaryotic ubiquitin-like protein on the Mycobacterium proteasomal ATPase targets substrates for degradation. Nat Struct Mol Biol. 2010; 17:1352-7. [PubMed: 20953180]

65. Festa RA, McAllister F, Pearce MJ, Mintseris J, Burns KE, Gygi SP, Darwin KH. Prokaryotic ubiquitin-like protein (Pup) proteome of Mycobacterium tuberculosis [corrected]. PLoS One. 2010; 5:e8589. [PubMed: 20066036]

66. Watrous J, Burns K, Liu WT, Patel A, Hook V, Bafna V, Barry CE 3rd, Bark S, Dorrestein PC. Expansion of the mycobacterial "PUPylome". Mol Biosyst. 2010; 6:376-85. [PubMed: 20094657]

67. Poulsen C, Akhter Y, Jeon AH, Schmitt-Ulms G, Meyer HE, Stefanski A, Stuhler K, Wilmanns M, Song YH. Proteome-wide identification of mycobacterial pupylation targets. Mol Syst Biol. 2010; 6:386. [PubMed: 20631680]

68. Gareau JR, Lima CD. The SUMO pathway: emerging mechanisms that shape specificity, conjugation and recognition. Nat Rev Mol Cell Biol. 2010; 11:861-71. [PubMed: 21102611]

69. MacMicking J, Xie QW, Nathan C. Nitric oxide and macrophage function. Annu Rev Immunol. 1997; 15:323-50. [PubMed: 9143691]

70. Lamichhane G, Raghunand TR, Morrison NE, Woolwine SC, Tyagi S, Kandavelou K, Bishai WR. Deletion of a Mycobacterium tuberculosis proteasomal ATPase homologue gene produces a slowgrowing strain that persists in host tissues. J Infect Dis. 2006; 194:1233-40. [PubMed: 17041849]

71. Sassetti CM, Boyd DH, Rubin EJ. Genes required for mycobacterial growth defined by high density mutagenesis. Mol Microbiol. 2003; 48:77-84. [PubMed: 12657046]

72. Gandotra S, Schnappinger D, Monteleone M, Hillen W, Ehrt S. In vivo gene silencing identifies the Mycobacterium tuberculosis proteasome as essential for the bacteria to persist in mice. Nat Med. 2007; 13:1515-20. [PubMed: 18059281] 
73. Gandotra S, Lebron MB, Ehrt S. The Mycobacterium tuberculosis proteasome active site threonine is essential for persistence yet dispensable for replication and resistance to nitric oxide. PLoS Pathog. 2010; 6:e1001040. [PubMed: 20711362]

74. Gottesman S. Proteolysis in bacterial regulatory circuits. Annu Rev Cell Dev Biol. 2003; 19:56587. [PubMed: 14570582]

75. Gur E, Biran D, Ron EZ. Regulated proteolysis in Gram-negative bacteria-how and when? Nat Rev Microbiol. 2011; 9:839-48. [PubMed: 22020261]

76. Collins GA, Tansey WP. The proteasome: a utility tool for transcription? Curr Opin Genet Dev. 2006; 16:197-202. [PubMed: 16503126]

77. Maciag A, Dainese E, Rodriguez GM, Milano A, Provvedi R, Pasca MR, Smith I, Palu G, Riccardi G, Manganelli R. Global analysis of the Mycobacterium tuberculosis Zur (FurB) regulon. J Bacteriol. 2007; 189:730-40. [PubMed: 17098899]

78. Serafini A, Boldrin F, Palu G, Manganelli R. Characterization of a Mycobacterium tuberculosis ESX-3 conditional mutant: essentiality and rescue by iron and zinc. J Bacteriol. 2009; 191:6340-4. [PubMed: 19684129]

79. Siegrist MS, Unnikrishnan M, McConnell MJ, Borowsky M, Cheng TY, Siddiqi N, Fortune SM, Moody DB, Rubin EJ. Mycobacterial Esx-3 is required for mycobactin-mediated iron acquisition. Proc Natl Acad Sci U S A. 2009; 106:18792-7. [PubMed: 19846780]

80. Nanamiya H, Akanuma G, Natori Y, Murayama R, Kosono S, Kudo T, Kobayashi K, Ogasawara N, Park SM, Ochi K, Kawamura F. Zinc is a key factor in controlling alternation of two types of L31 protein in the Bacillus subtilis ribosome. Mol Microbiol. 2004; 52:273-83. [PubMed: 15049826]

81. Natori Y, Nanamiya H, Akanuma G, Kosono S, Kudo T, Ochi K, Kawamura F. A fail-safe system for the ribosome under zinc-limiting conditions in Bacillus subtilis. Mol Microbiol. 2007; 63:294307. [PubMed: 17163968]

82. Pruteanu M, Baker TA. Proteolysis in the SOS response and metal homeostasis in Escherichia coli. Res Microbiol. 2009; 160:677-83. [PubMed: 19747971]

83. Festa RA, Jones MB, Butler-Wu S, Sinsimer D, Gerads R, Bishai WR, Peterson SN, Darwin KH. A novel copper-responsive regulon in Mycobacterium tuberculosis. Mol Microbiol. 2011; 79:13348. [PubMed: 21166899]

84. Gold B, Deng H, Bryk R, Vargas D, Eliezer D, Roberts J, Jiang X, Nathan C. Identification of a copper-binding metallothionein in pathogenic mycobacteria. Nat Chem Biol. 2008; 4:609-16. [PubMed: 18724363]

85. Samanovic MI, Ding C, Thiele DJ, Darwin KH. Copper in microbial pathogenesis: meddling with the metal. Cell Host Microbe. 2012; 11:106-15. [PubMed: 22341460]

86. Prentice AM, Ghattas H, Cox SE. Host-pathogen interactions: can micronutrients tip the balance? J Nutr. 2007; 137:1334-7. [PubMed: 17449601]

87. Humbard MA, Miranda HV, Lim JM, Krause DJ, Pritz JR, Zhou G, Chen S, Wells L, MaupinFurlow JA. Ubiquitin-like small archaeal modifier proteins (SAMPs) in Haloferax volcanii. Nature. 2010; 463:54-60. [PubMed: 20054389]

88. Delley CL, Striebel F, Heydenreich FM, Ozcelik D, Weber-Ban E. Activity of the mycobacterial proteasomal ATPase Mpa is reversibly regulated by pupylation. J Biol Chem. 2012; 287:7907-14. [PubMed: 22210775] 


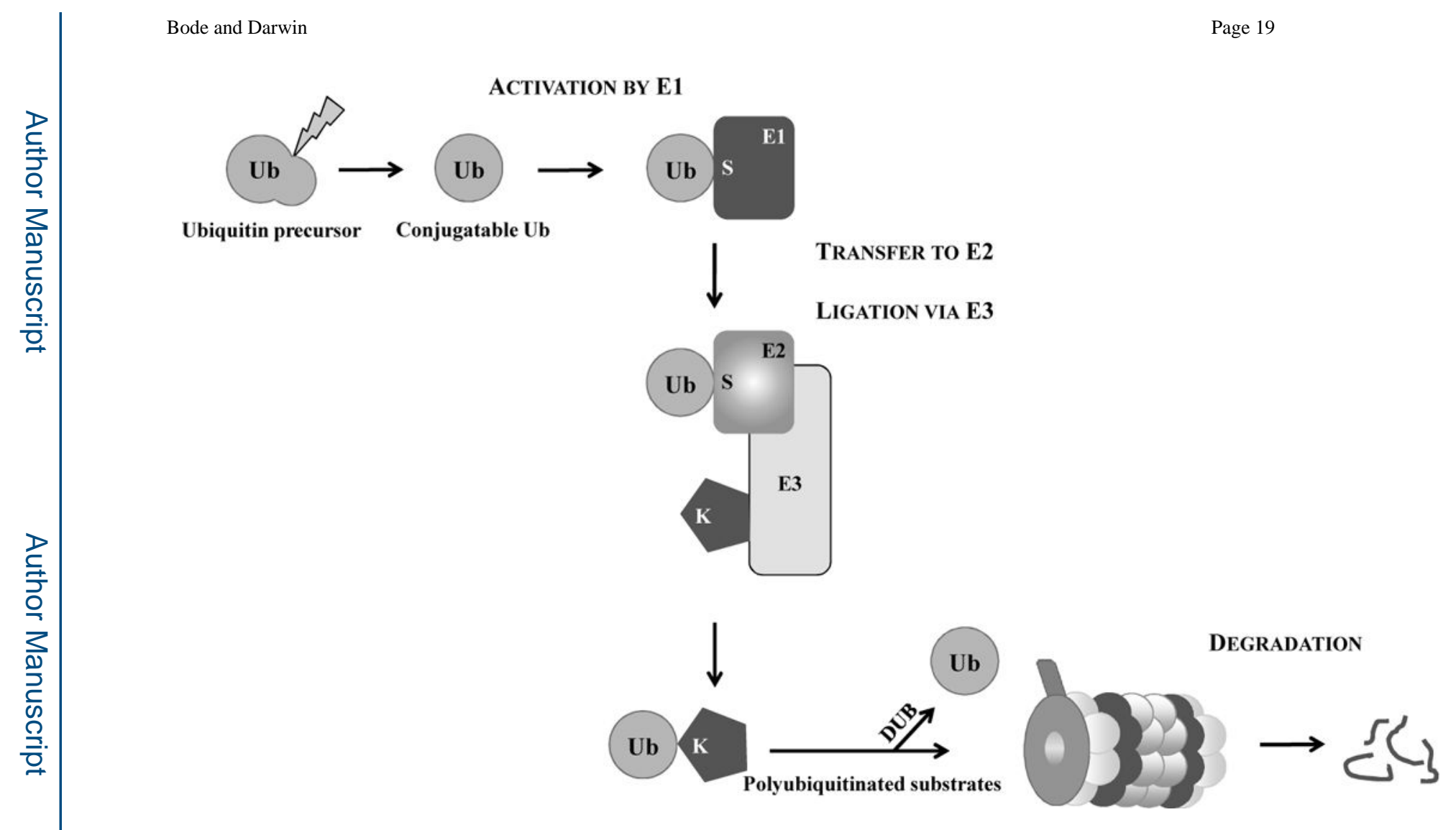

Figure 1. Eukaryotic ubiquitin-proteasome system

Ubiquitin (Ub) precursors are processed to expose a C-terminal di-glycine motif. The conjugation-competent $\mathrm{Ub}$ is adenylated and subsequently bound in a high-energy thioester bond by the E1-activating enzyme. Ub is then transferred to the catalytic cysteine of an E2conjugating enzyme. Ub can be ligated to substrates with the help of E3-ligases. Typically, tetra-Ub chains linked at lysine 48 are recognized by the $26 \mathrm{~S}$ proteasome. Deubiquitylases can remove $\mathrm{Ub}$ from substrates. 
DEAMIDATION
LIGATION

DEPUPYLATION

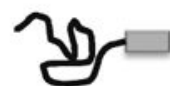

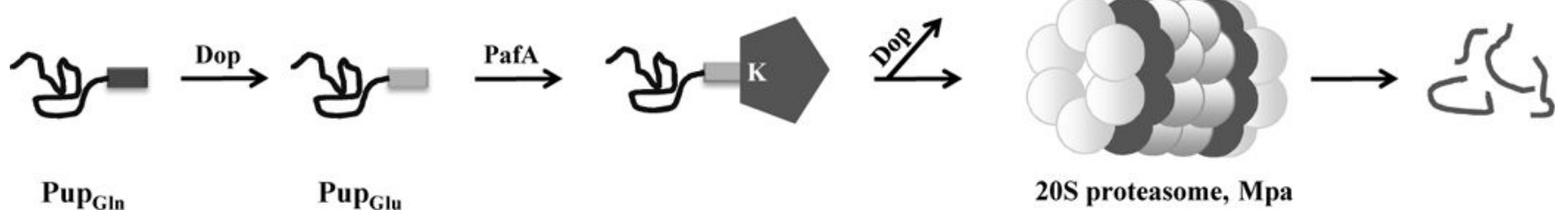

Figure 2. Mycobacterial pupylation pathway

Pup is deamidated at the C-terminal glutamine by Dop (deamidase of Pup). The Pup ligase PafA phosphorylates the C-terminal $\gamma$-carboxylate of Pup and then conjugates Pup to lysine residues of target proteins via an isopeptide bond. The mycobacterial proteasome and its cognate ATPase Mpa degrade pupylated proteins. Dop also acts as a depupylase, allowing for Pup to be recycled. 


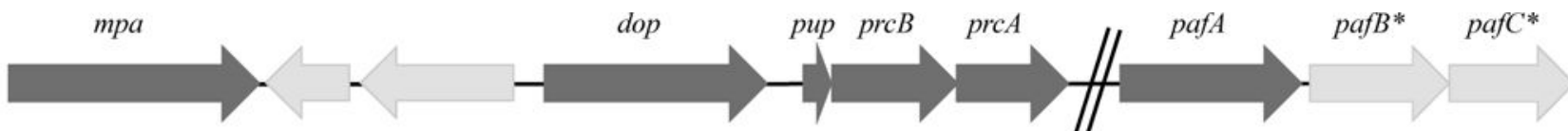
H37Rv Rv2115c
CDC155 MT2175
mc $^{2} 155$ MSMEG_3902

Rv2112c

Rv2111c Rv2110c Rv2109c

Rv2097c

$\begin{array}{llll}\text { MT2172 } & \text { MT2171 } & \text { MT2170 } & \text { MT2169 }\end{array}$

MT2158

$$
\begin{gathered}
\text { MSMEG_3897 } \\
\text { MSMEG_3896 }
\end{gathered} \stackrel{\text { MSMEG_3894 }}{\text { MSMEG_3890 }}
$$

Figure 3. Genomic organization of PPS genes in mycobacteria

Gene data are from http://tuberculist.epfl.ch/. * pafB and pafC are cotranscribed with pafA in $M t b$ H37Rv (46). However, no apparent contribution to pupylation has been demonstrated for PafB or PafC (40). 


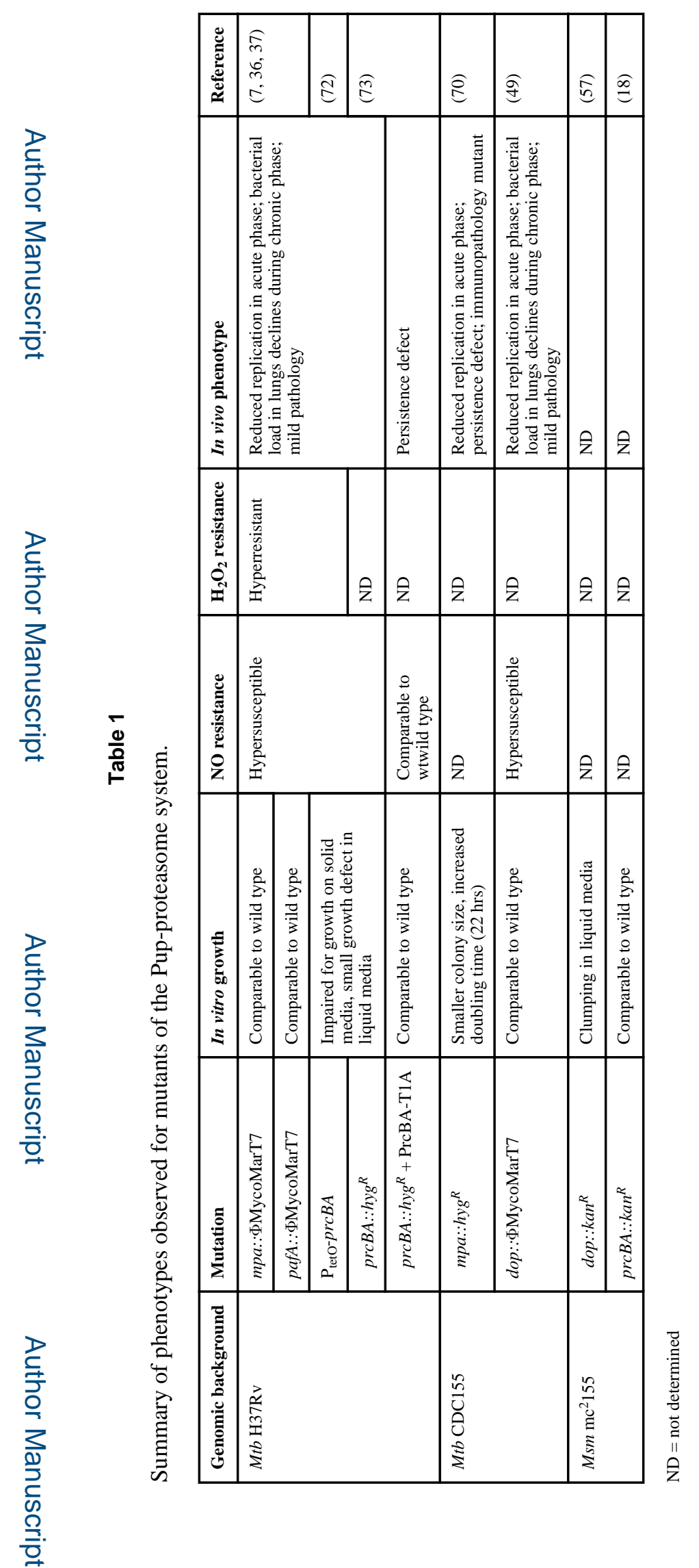

Microbiol Spectr. Author manuscript; available in PMC 2014 November 15. 